\title{
Management of pregnant women infected with Ebola virus in a treatment centre in Guinea, June 2014
}

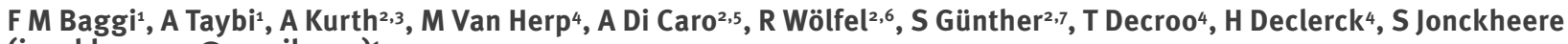

(jonckheeresy@gmail.com) ${ }^{1}$

1. Médecins Sans Frontières - Operational Centre Brussels, Guéckedou, Guinea

2. European Mobile Laboratory - Hamburg, Germany

3. Robert Koch Institute - Berlin, Germany

4. Médecins Sans Frontières - Operational Centre Brussels, Medical Department, Brussels, Belgium

5. L. Spallanzani National Institute for Infectious Diseases - Rome, Italy

6. Bundeswehr Institute of Microbiology - Munich, Germany

7. Bernhard-Nocht-Institute for Tropical Medicine, WHO Collaborating Centre for Arboviruses and Hemorrhagic Fever Reference and Research - Hamburg, Germany

Baggi FM, Taybi A, Kurth A, Van Herp M, Di Caro A, Wölfel R, Günther S, Decroo T, Declerck H, Jonckheere S. Management of pregnant women infected with Ebola virus in a treatment centre in Guinea, June 2014. Euro Surveill. 2014;19(49):pii=20983. Available online: http://www.eurosurveillance.org/ViewArticle. aspx?Articleld $=20983$

We report two cases of confirmed Ebola virus disease in pregnant women, who presented at the Médecins Sans Frontières Ebola treatment centre in Guéckédou. Despite the very high risk of death, both pregnant women survived. In both cases the critical decision was made to induce vaginal delivery. We raise a number of considerations regarding the management of Ebola virus-infected pregnant women, including the place of amniocentesis and induced delivery, and whether certain invasive medical acts are justified.

We report two cases of confirmed Ebola virus disease (EVD) in pregnant patients who presented and were treated at the Médecins Sans Frontières (MSF) Ebola treatment centre in Guéckedou. We also raise a number of considerations regarding the role of amniocentesis and induced delivery in the management of pregnant women with EVD.

\section{Description of the cases}

\section{Case one}

\section{Initial presentation}

At the beginning of June 2014, a woman in her late $20 \mathrm{~S}$ at seven months gestation presented at the Ebola treatment centre in Guékedou, Guinea, with a history of seven days of asthenia, fever (self-reported), and vomiting. Her past obstetrical history included six vaginal deliveries and no abortions. On admission, physical examination revealed a temperature of $37.1^{\circ} \mathrm{C}$, mild dehydration and the patient reported fetal movement. The Ebola virus (EBV) test (real-time reverse transcription-polymerase chain reaction (RT-PCR)) was positive.

Clinical course and management

On the same day (day o), the woman was admitted to the EBV treatment/isolation unit where she immediately started receiving supportive treatment, including Ringer's lactate, antipyretics, ceftriaxone (2 g/day), metoclopramide and omeprazole. The woman responded well to this supportive treatment and by day six of her admission, she was free from symptoms, and reported continuously fetal movements. On day eight and 10 , the results of the EBV tests came back negative, and the woman was considered cured. The woman remained in the unit for further monitoring.

On day 11 , the woman's temperature rose to $38^{\circ} \mathrm{C}$, and further examination revealed that fetal movements and heartbeat had stopped. Cervical examination showed no uterine contractions, no cervical dilation, no blood or other discharge. Intravenous metronidazole was added for suspected chorioamnionitis. To evaluate the possibility of maternal-to-fetal EBV transmission, an amniocentesis was performed. The clear-coloured amniotic fluid contained a high Ebola viral load (corresponding to a real-time RT-PCR cycle threshold (CT) value of 21.29).

On day 15, the patient was afebrile. An assisted delivery was organised to take place in the high-risk zone of the treatment centre. Labour induction with misoprostol resulted in a vaginal delivery of a stillborn male fetus (first degree maceration). The placenta was complete. No episiotomy was required, uterine bimanual massage, oxytocin (10 Units intravenous) and ergometrine (one vial of $0.2 \mathrm{mg}$ intramuscular) helped obtaining normal uterine retraction and prevented any excessive post-partum bleeding. The samples from the placenta (maternal and fetal side), meconium, and the fetus (intra-cardiac aspiration, throat swab, ear swab, umbilical cord) were EBV positive (Table). The patient was afebrile after delivery, and was discharged on day 18. A seven-days post-natal consultation (PNC) showed a normal evolution. 
Test results from maternal and fetal samples taken from two pregnant patients during their stay at the Ebola treatment centre, Guéckédou, Guinea, June 2014

\begin{tabular}{|c|c|c|c|c|}
\hline Timeline & Specimen type & $\begin{array}{l}\text { Ebola virus load } \\
\text { result (CT value) }\end{array}$ & $\begin{array}{l}\text { Semi-quantitative } \\
\text { viral load result }\end{array}$ & Other results \\
\hline \multicolumn{5}{|l|}{ Patient 1} \\
\hline Day o (admission) & Blood (mother) & Positive (21.29) & +++ & Malaria negative \\
\hline Day 8 & Blood (mother) & Negative (-) & - & $\begin{array}{l}\text { IgG positive }(\geq 1: 1,280) \\
\text { IgM positive }(\geq 1: 320)\end{array}$ \\
\hline Day 10 & Blood (mother) & Negative (-) & - & - \\
\hline Day 12 & Amniotic fluid (amniocentesis) & Positive (23.31) & +++ & - \\
\hline Day 15 & $\begin{array}{l}\text { Amniotic fluid (fetal mouth } \\
\text { swab) }\end{array}$ & Positive (21.41) & +++ & - \\
\hline Day 15 & Amniotic fluid (fetal ear swab) & Positive $(24.78)$ & +++ & - \\
\hline Day 15 & Placenta (fetal side) & Positive $(24,12)$ & +++ & - \\
\hline Day 15 & Placenta (maternal side) & Positive $(19,23)$ & +++ & - \\
\hline Day 15 & Fetal blood - sample 1 & Positive $(16,13)$ & +++ & - \\
\hline Day 15 & Fetal blood - sample 2 & Positive (23.6) & +++ & - \\
\hline Day 15 & Fetal meconium (anus swab) & Positive $(20,32)$ & +++ & - \\
\hline Day 18 & Blood (mother) & Negative (-) & - & - \\
\hline \multicolumn{5}{|l|}{ Patient 2} \\
\hline Day $1^{a}$ & Blood (mother) & Positive (26.46) & ++ & Malaria positive \\
\hline Day 7 & Blood (mother) & Positive $(25,43)$ & ++ & - \\
\hline Day 11 & Amniotic fluid (mouth swab) & Positive (24.10) & +++ & - \\
\hline Day 11 & Amniotic fluid (fetal ear swab) & Positive (28.82) & ++ & - \\
\hline Day 11 & Placenta (fetal side) & Positive (14.22) & +++ & - \\
\hline Day 11 & Placenta (maternal side) & Positive (19.98) & +++ & - \\
\hline Day 11 & Fetal meconium (anus swab) & Negative (-) & - & - \\
\hline Day 16 & Blood (mother) & Negative (-) & - & - \\
\hline Day 18 & Blood (mother) & Negative (-) & - & - \\
\hline
\end{tabular}

CT: cycle threshold; NA: not available; RT-PCR: reverse transcription-polymerase chain reaction.

a Patient 2 first presented at the treatment centre in the afternoon (day o) so the result of Ebola virus testing was available the next day (day 1).

Real-time RT-PCR was performed with the Smart Cycler. The obtained CT values correspond with the accumulation of the fluorescent signal and are inversely proportional with the viral load. CT values are classified in subsequent categories of $0-25,25-35$ and $35-40$ and correspond with,+++++ and + results.

When the real-time RT-PCR was negative this is indicated for the viral load by a - result.

\section{Case two}

\section{Initial presentation}

In mid-June 2014 a primipara in her early 20 s at seven months gestation presented with a history of five days of arthralgia, asthenia, diarrhoea, fever (self-reported) and headache. The patient presented with a history of grade III female genital mutilation (FGM). On admission, the patient had a temperature of $38.4^{\circ} \mathrm{C}$, and reported fetal movements and no contractions.

Clinical course and management

On the next day (day 1), the EBV test and malaria rapid test were positive. The patient's fever worsened $\left(39.5^{\circ} \mathrm{C}\right)$, and she had an onset of haematuria and cough. The patient reported fetal movements had stopped. Supportive treatment included intravenous ampicillin and metronidazole for a possible chorioamnionitis, as well as intravenous artesunate (malaria treatment) and Ringer's lactate.

On day five, the patient's systolic blood pressure dropped to $60 \mathrm{mmHg}$ (norm: 90-119) and additional fluids were intravenously administered. On day eight, the patient presented with symmetrical oedema of the lower extremities. Obstetrical examination revealed a hypertonic uterus, transverse or breech presentation, no fetal heartbeat, cervical dilation of one centimeter in diameter, and no discharge. Despite the risk, ketamine anaesthesia was provided, external version manoeuvres were performed, and the fetus was rendered in cephalic presentation. 
On day 10, the patient was disoriented and presented with anasarca. On the morning of day 11, the patient was found unconscious, with the fetal head intravaginal. Ketamine was administered, an episiotomy was performed, and a male stillborn fetus was delivered vaginally. The placenta was complete. Urinary retention complicated uterine retraction, and uterine bi-manual massage was employed together with the administration of oxytocin (10 U) and ergometrine (1 vial of $0.2 \mathrm{mg}$ ). Post-partum haemorrhage only stopped after repeatedly (five times) packing the uterus with gauze. Due to the FGM, bladder catheterisation was unsuccessful. Urine was aspirated through a suprapubic bladder paracentesis (the urine was not tested for EBV). A final vaginal and uterine exploration showed no further complications. The samples from the placenta (maternal and fetal side) and the fetus (throat swab, ear swab) were EBV positive. The sample from the meconium was negative (Table). No pericardial puncture was performed.

On day 12 , the patient regained consciousness, and spontaneous diuresis resumed after a single dose dexamethasone injection. The patient had a temperature of $40^{\circ} \mathrm{C}$. Gentamicin was added to the treatment. Over the next six days the patient improved clinically. On day 17, the patient was afebrile. On day 16 and 18 , EBV tests were negative and the patient was considered cured. The patient was discharged on day 19. She did not attend her scheduled appointment seven-days PNC.

\section{Ebola virus outbreak in West Africa}

In March 2014, an EVD outbreak was declared in Guéckedou, Guinea, following which it spread to Liberia, Sierra Leone, Nigeria, Senegal and Mali $[1,2]$. The viral strain responsible for the current outbreak has been identified as the Zaire strain, a particularly virulent strain associated with mortality rates as high as 90\% [1]. Overall, by 21 November 2014, 15,351 individuals have become infected and 5,459 of these have died. Among those infected, 588 were healthcare workers and 377 of these have died [2]. Patients with EVD generally present with a history of contact with another person with EVD and an abrupt onset of a nonspecific febrile syndrome. A systemic inflammatory response can cause multiple organ failure and shock $[3,4]$. Pregnant women are reported to be at higher risk to die [5].

Since the onset of the outbreak in Guinea, MSF has set up and is running six Ebola treatment centres - including one in Guékédou where the outbreak began.

\section{Discussion}

There are very few studies reporting on maternal and fetal outcomes of pregnant women infected with EBV. We report on two cases of pregnant women infected with EBV in Guinea. Despite pregnant women being at higher risk of more severe disease and mortality [5], both women survived. Both fetuses unfortunately died in utero. This case report raises a number of important points for discussion regarding the management of pregnant women infected with EBV.

Although our findings are based on two cases only, they depict a more positive picture of the maternal outcomes of EVD during pregnancy. In both cases the delivery occurred during the healing phase, when the EBV viraemia in the pregnant woman was controlled, and when clotting had probably returned to normal. During previous outbreaks also caused by the Zaire strain, such as in the Democratic Republic of the Congo (DRC) 1976 Yambuku outbreak, only nine (11\%) of 82 EBV infected pregnant women were reported to have survived [6]. Similarly, during another EBV outbreak in the DRC 20 years later (1995 in Kikwit) only one (7\%) of 15 pregnant women was reported to have survived, and EBV-infected pregnant women had a notably higher mortality rate (93\%) than non-pregnant EBV-infected women (70\%) [5]. For the current outbreak, data on pregnancy are not routinely reported so overall figures on the survival of pregnant women and their unborn children or neonates are not available at this point.

Despite the two women described in this report surviving, in both cases the fetus died in utero. There is not much chance for the fetus to survive EBV infection. A massive infection of the fetus is likely to occur through the placenta. Furthermore maternal immunoglobulins $M$ are poorly transported through placental villi and the fetal secretory immune system starts producing immunoglobulin $M$ around the 2oth week of gestation [7]. Fetal and neonate mortality was equally reported high in other outbreaks. In Yambuku, 11 live neonates were born to EBV-infected women and all died within 19 days [6]. In Yambuku and Kikwit, abortion occurred among 19 of $82(23 \%)$ and 10 of $15(67 \%)$ infected pregnant women respectively $[5,6]$. In Kikwit the only surviving pregnant patient had an abortion [5]. In Yambuku, one of the nine survivors aborted in the treatment centre. Abortions occurred spontaneously, likely because of fetal death due to EBV infection. Unfortunately the pregnancy outcomes of the other pregnant EVD survivors were not reported [6].

This is the first description of the use of amniocentesis to determine the presence of intrauterine EBV infection. In the case of the first patient, despite her having an undetectable viral load and declared cured before the demise of the fetus, a subsequent amniocentesis revealed a high viral load in the amniotic fluid. For this reason, a vaginal delivery was arranged to take place in the high-risk zone of the EVD treatment centre. In the absence of the amniocentesis, the recovered EBV negative patient might have been referred to the local maternity for delivery, exposing the maternity staff to a very high risk of EBV infection. Alternatively, she might have had a spontaneous abortion at home with potential risk of subsequent transmission of EBV to household contacts. In the second case, an emergency 
delivery was required and performed at the EVD treatment centre after the patient was found in shock, with intra-vaginal fetal head. This delivery also took place in the high-risk zone of the centre and episiotomy was justified due to the grade III FGM. Post-partum, an amniotic fluid sample was taken from the dead fetus through an oral swab and yielded a high EBV viral load.

For both cases, the assisted delivery occurred a few days after fetal movement had reportedly stopped. As these were the first induced deliveries of EBV infected pregnant women, careful planning had to be considered as well precautionary measures, given the high risk of nosocomial transmission to healthcare workers [3]. Moreover, based on previous reports $[5,6]$, it was also taken into account that spontaneous abortions could occur shortly after fetal death, limiting the need for invasive procedures, and reducing the risk to healthcare staff.

During both deliveries, strict barrier nursing techniques were used. Full protective equipment included scrubs, waterproof overall, apron, boots, N95 masks, head cover, goggles, a double pair of gloves and armlength gynaecological gloves (three layers of gloving). Absorbent pads were laid underneath the patients to absorb a maximal amount of fluids. Two pads were laid over the abdomen and the perineal region to limit splashing. Biomedical waste was gathered in the immediate proximity of the patient, and regularly sprayed with $0.5 \%$ chlorine solution. None of the five healthcare workers who were present during the deliveries reported here became infected.

These two case presentations raise a number of considerations regarding the management of pregnant women infected with EBV including the role of amniocentesis and induced delivery, and whether certain invasive medical procedures are justified, despite the inherent risk for healthcare workers.

In conclusion, our case report adds to the scarce body of literature on the outcomes of pregnant women infected with EBV. We also highlight some important considerations in the management of such patients and describe, for the first time, the use of amniocentesis to detect fetal infection with EBV.

\section{Acknowledgements}

We would like to thank Marguerite Ouamouno, Pauline Yombouno, Adèle Millimouno and all MSF staff from Guékédou for their support in taking care of these and many other patients. We acknowledge Rosa Crestani and Katherine Harries for their critical review of the manuscript.
Authors' contribution

Fernanda Mendez Baggi, Aicha Taybi, Andreas Kurth, and Sylvie Jonckheere collected data in the MSF Ebola treatment centre in Guéckedou, and wrote the first draft. Michel Van Herp, Antonino Di Caro, Roman Wölfel, Stephan Günther, Hilde Declerck, and Tom Decroo all reviewed the first draft and final version of the paper, and their comments were incorporated.

\section{References}

1. Baize S, Pannetier D, Oestereich L, Rieger T, Koivogui L, Magassouba NF, et al. Emergence of Zaire Ebola Virus Disease in Guinea. N Engl J Med. 2014;371(15):1418-25. http://dx.doi. org/10.1056/NEJMoa1404505

2. World Health Organization (WHO). Ebola Response Roadmap. Situation Report Update. 21 November 2014. Geneva: WHO; 2014. Available from: http://apps.who.int/iris/ bitstream/10665/144117/1/roadmapsitrep_21Nov2014_eng.pdf

3. Feldmann H, Geisbert TW. Ebola haemorrhagic fever. Lancet. 2011;377(9768):849-62. http://dx.doi.org/10.1016/S01406736(10)60667-8 PMID:21084112

4. Leroy EM, Gonzalez JP, Baize S. Ebola and Marburg haemorrhagic fever viruses: major scientific advances, but a relatively minor public health threat for Africa. Clin Microbiol Infect. 2011;17(7):964-76. http://dx.doi.org/10.1111/j.14690691.2011.03535.x PMID:21722250

5. Mupapa K, Mukundu W, Bwaka MA, Kipasa M, De Roo A Kuvula K, et al. Ebola hemorrhagic fever and pregnancy. J Infect Dis. 1999;179(s1) Suppl 1;S11-2. http://dx.doi. org/10.1086/514289 PMID:9988157

6. Johnson KM. Ebola haemorrhagic fever in Zaire, 1976. Bull World Health Organ. 1978;56(2):271-93. PMID:307456

7. Zusman I, Gurevich P, Ben-Hur H. Two secretory immune systems (mucosal and barrier) in human intrauterine development, normal and pathological (Review). Int J Mol Med. 2005;16(1):127-33. PMID:15942689 